\title{
Descortezado en forestales producido por la ardilla introducida Callosciurus erythraeus (Sciuridae) en Argentina
}

\author{
Bark stripping caused by the introduced squirrel \\ Callosciurus erythraeus (Sciuridae) in Argentina
}

\author{
Paula A Pedreira ${ }^{\mathrm{a}, \mathrm{b} *}$, Eduardo Penon ${ }^{\mathrm{c}}$, Mariela Borgnia ${ }^{\mathrm{a}, \mathrm{b}}$ \\ ${ }^{a}$ Universidad Nacional de Luján, Departamento de Cs. Básicas, Buenos Aires, Argentina.

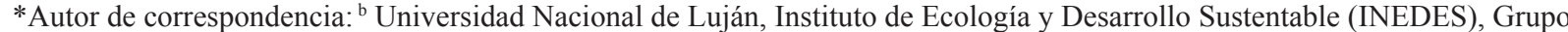 \\ Ecología de Mamíferos Introducidos, CONICET, Buenos Aires, Argentina, tel.: 011-15-61683730, anabelpedreira@gmail.com \\ c Universidad Nacional de Luján, Departamento de Tecnología y Dasonomía, Instituto de Ecología y Desarrollo Sustentable (INEDES), \\ Universidad Nacional de Luján-CONICET, Buenos Aires, Argentina.
}

\begin{abstract}
SUMMARY
An assessment of bark stripping damage caused by the introduced red-bellied squirrel (Callosciurus erythraeus) was carried out in the area where this rodent is currently established in Argentina. The study was carried out in four forested areas, on 38 plots of 150 and $250 \mathrm{~m}^{2}$, where damaged species, type and intensity of the damage were recorded. Squirrel severely debarked 12 forest species and $57 \%$ of sampled trees showed wounds below $5 \mathrm{~m}$. Results showed that the red-bellied squirrel can be considered a harmful species for forests in the study area and could be a potential problem to other regions of Argentina if it continues its invading dispersal. The implications to forest production were discussed.
\end{abstract}

Key words: red-bellied squirrel, invasive species, tree damage, forest pest.

\section{RESUMEN}

En este trabajo se describe el daño ocasionado en diferentes árboles por un mamífero exótico introducido en Argentina, la ardilla de vientre rojo (Callosciurus erythraeus), en el principal foco de invasión del país. Se trabajó en cuatro lugares forestados, donde se registraron las especies dañadas, el tipo e intensidad del daño, en 38 parcelas de entre 150 y $250 \mathrm{~m}^{2}$. Las ardillas afectaron una gran cantidad de individuos y especies forestales, varias de ellas de importancia comercial. En 12 especies se registraron árboles con elevados niveles de daño observándose que en promedio el $57 \%$ de los individuos presentó heridas por debajo de los 5 m. Los resultados revelaron que la ardilla de vientre rojo resulta una especie perjudicial en su área de influencia para muchas especies forestales y puede ser una amenaza para otras regiones forestales del país si continúa expandiéndose; se discuten las implicancias de su impacto en términos de la producción forestal.

Palabras clave: ardilla de vientre rojo, especies invasoras, descortezamiento, plaga forestal.

\section{INTRODUCCIÓN}

$\mathrm{La}$ ardilla de vientre rojo Callosciurus erythraeus (Pa1las) es de origen asiático y por sus hábitos de consumo y su actividad de descortezado se ha convertido en un serio problema, para la producción de frutales y madera, al igual que otras especies de ardillas (Mora-Ascencio et al. 2010, Yin et al. 2010, Bertolino y Lurz 2013).

Luego de 40 años de introducida en el partido de Luján (Buenos Aires, Argentina), la ardilla de vientre rojo es considerada una especie invasora que se expande por causas naturales y antrópicas y ocasiona diversos impactos en el ecosistema (Aprile y Chicco 1999, Gozzi et al. 2013, Guichón et al. 2015, Messetta et al. 2015). Hay evidencias de que estas ardillas descortezan diversos árboles (Guichón et al. 2005, Zubelzu et al. 2015), pero en Argentina, su impacto, no ha sido cuantificado. Las políticas gubernamentales actuales incentivan la producción forestal, siendo Eucalyptus, Pinus, Populus y Salix los géneros más implantados (Núñez 2007). Es importante poder conocer y cuantificar los daños que ocasiona esta ardilla, para predecir y prevenir su dispersión hacia nuevas áreas, o mitigar dichos daños (Smallwood y Salmon 1992).

Bajo la hipótesis de que la ardilla $C$. erythraeus se comporta como una nueva especie perjudicial en Argentina, el objetivo de este trabajo es caracterizar y cuantificar el daño por descortezamiento que produce este mamífero exótico en diferentes especies forestales dentro del principal foco de invasión en el país. 


\section{MÉTODOS}

El estudio fue realizado durante el invierno de 2014 en cuatro áreas forestales con vegetación natural o implantada en su mayoría de especies exóticas dentro del partido de Luján: 1) Club "El Timón": 34 35'35,68" S, $59^{\circ}$ $11^{\prime} 13,43^{\prime \prime} \mathrm{O}$, con vegetación de ribera mixta, predominando casuarina (Casuarina sp.), acacia negra (Gleditsia triacanthos), ligustro (Ligustrum lucidum), sauce (Salix sp.), álamo (Populus spp.) y olmo (Ulmus spp.); 2) Establecimiento forestal "Liebres Fue" : $34^{\circ} 36^{\prime} 12,1^{\prime \prime} \mathrm{S}, 59^{\circ}$ $11^{\prime} 32,1^{\prime \prime}$ O, con rodales de eucalipto blanco (Eucalyptus dunnii) de 5 y 18 años de edad, pino elliotti (Pinus elliottii), de 20 años de edad, y roble europeo (Quercus robur de 30 años de edad; 3) Predio Hospital Cabred: 34 30'11" S, $59^{\circ} 04^{\prime} 18^{\prime \prime} \mathrm{O}$, con lotes de Q. robur, cedro (Cedrus deodara), lotes mixtos de eucaliptos tereticornis (Eucalyptus tereticornis)/G. triacanthos y de ciprés (Cupressus sp.)/ Casuarina sp. y, 4) "Arboretum" de la Universidad Nacio-

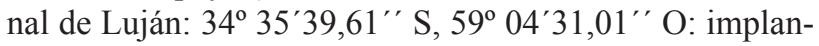
tado hace 31 años, con álamos (Populus spp.), Eucalyptus spp., roble europeo y castaños (Castanea sativa) (figura 1).

Se evaluaron 38 parcelas circulares en cuatro (4) áreas forestales: 1) siete parcelas de $150 \mathrm{~m}^{2}$ con $12-33$ árboles por parcela; 2) 12 parcelas de $150-250 \mathrm{~m}^{2}$ con 2-18 árboles por parcela; 3) seis parcelas de $150 \mathrm{~m}^{2}$ con 9-31 árboles por parcela y 4) 13 parcelas de $150 \mathrm{~m}^{2}$ con 5-23 árboles por parcela) distribuidas aleatoriamente y separadas entre sí con un mínimo de $100 \mathrm{~m}$, cubriendo un área efectiva de $6.300 \mathrm{~m}^{2}$.

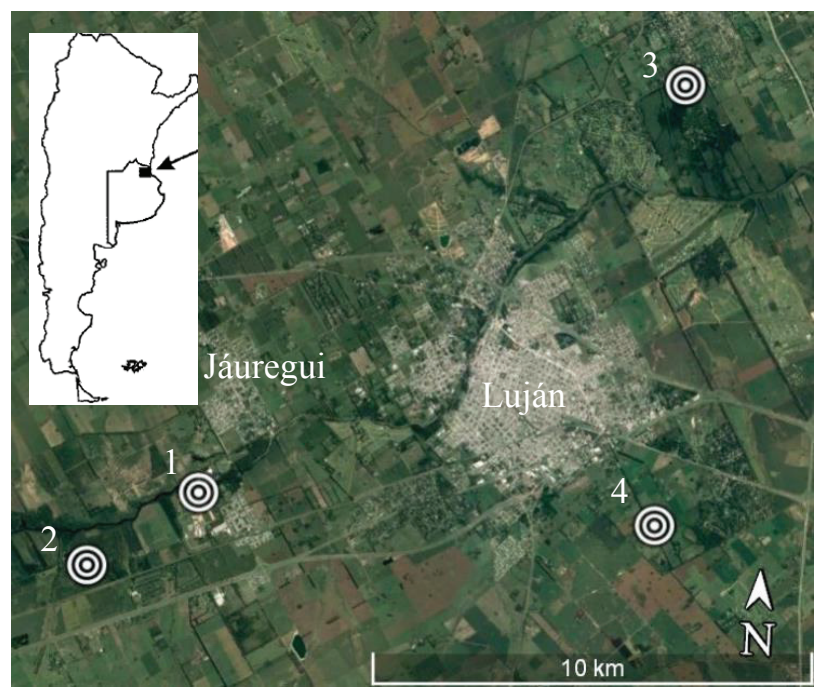

Figura 1. Área de estudio dentro del Partido de Luján (Buenos Aires, Argentina): 1) Club "El Timón", 2) Establecimiento "Liebres Fue", 3) Predio forestal "Hospital Cabred" y 4) "Arboretum" de la Universidad Nacional de Luján.

Study area in Lujan District (Buenos Aires, Argentina): 1) 'El Timón' recreational club, 2) 'Liebres Fue' productive land, 3) 'Hospital Cabred' forest land and 4) 'Arboretum' of National University of Luján.
En cada parcela se registró: especie arbórea, altura del árbol, diámetro a la altura del pecho (DAP), presencia y ubicación del daño (tronco o rama), altura e intensidad del daño (proporción de la longitud total del tronco afectada o proporción de la totalidad de las ramas afectadas) según seis categorías $(1-5 \%, 6-10 \%, 11-25 \%, 26-50 \%, 51$ - $75 \%, 76-100 \%$ ), y forma de las heridas. Se realizó un análisis estadístico descriptivo de los datos considerando todos los árboles, se analizaron las frecuencias para evaluar la intensidad y altura mínima de daño, y se comparó con la prueba t de Student posibles diferencias en la altura y DAP de individuos descortezados y no descortezados.

\section{RESULTADOS}

Las ardillas descortezaron el 40,6 \% del total de árboles $(n=542)$, y el $88 \%$ de las especies registradas $(n=24)$ (cuadro 1). Las especies con más del $50 \%$ de sus individuos dañados fueron P. elliottii, C. sativa, Casuarina sp., Ulmus spp., C. deodara, Fraxinus sp., Salix babylonica, Morus nigra y Eucalyptus spp. (E. cinerea, E. dunnii, E. camaldulensis) siendo variable el número de individuos de cada especie.

Las heridas se localizaron en el tronco, ramas o ambos, según la especie considerada. Se registraron dos tipos principales de heridas, pudiendo ambas estar presentes en los mismos árboles: 1) las longitudinales (con área variable, en sentido paralelo a la rama o tronco), presentes en todas las especies dañadas, y 2) heridas transversales (perpendiculares a la rama o tronco, que pueden en algunos casos anillarlo), registradas en siete especies arbóreas, siendo más frecuentes que las longitudinales en E. camaldulensis y Casuarina sp. En las heridas longitudinales recientes pudo observarse la corteza deshilachada (figura 2).

La intensidad del daño entre los individuos de una misma especie fue variable (cuadro 1). En 12 especies se registraron individuos con intensidades altas (categoría 5 o 6) en tronco o rama. Dentro de las especies de importancia comercial, por ejemplo, en robles, en más del $50 \%$ de los individuos alcanzó una intensidad de 4 y 5 en tronco y rama (figura 3A). En pino, el $50 \%$ de los árboles estuvo afectado a nivel de tronco, con una intensidad entre 3 y 4 (figura 3B). En salicáceas, la intensidad de daño fue mayor a nivel de tronco, alcanzando la categoría 3 en el $70 \%$ de los individuos (figura 3C). En eucaliptos, casi un $5 \%$ de los árboles fue dañado con categoría 5 (figura 3D).

En cuanto a la altura del daño, en el $57 \%$ de los árboles se registró en troncos por debajo de los $5 \mathrm{~m}$ (figura 4A) y para $E$. dunnii el $64 \%$ de los descortezados presentaron heridas por debajo de los $3 \mathrm{~m}$ de altura (figura 4B). En esta especie se observaron daños que originaron quebraduras con el consiguiente descope del individuo y posterior rebrote en la zona dañada.

$\mathrm{Al}$ comparar los árboles dañados $(\mathrm{n}=220)$ y no dañados $(\mathrm{n}=321)$, los individuos descortezados presentaron un DAP significativamente mayor ( $\overline{\mathbf{X}}=0,254 \mathrm{~m}$ ) que los no 
Cuadro 1. Porcentaje de individuos descortezados, ubicación del daño, forma de la herida, y rango de la intensidad del daño por especie. Percentage of debarked trees, location, shape and severity of wounds by species.

\begin{tabular}{|c|c|c|c|c|c|c|c|c|c|}
\hline \multirow[b]{2}{*}{ Especies de árboles } & \multirow[b]{2}{*}{$\begin{array}{l}\mathrm{N}^{\circ} \text { total de } \\
\text { individuos }\end{array}$} & \multirow{2}{*}{$\begin{array}{c}\% \text { de } \\
\text { individuos } \\
\text { dañados }\end{array}$} & \multicolumn{3}{|c|}{$\begin{array}{l}\text { Ubicación del daño } \\
\text { (\% de individuos) }\end{array}$} & \multicolumn{2}{|c|}{$\begin{array}{l}\text { Forma de la herida } \\
\text { (\% de individuos) }\end{array}$} & \multicolumn{2}{|c|}{$\begin{array}{c}\text { Rango de la intensidad } \\
\text { de daño (\%) }\end{array}$} \\
\hline & & & $\begin{array}{l}8 \\
\stackrel{0}{0} \\
ت\end{array}$ & 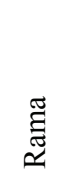 & 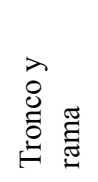 & 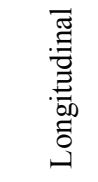 & 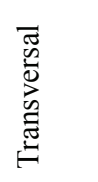 & $\begin{array}{l}8 \\
\stackrel{0}{0} \\
\stackrel{0}{H}\end{array}$ & $\begin{array}{l}\mathscr{\sharp} \\
\text { ॠ్ } \\
\approx\end{array}$ \\
\hline Araucaria bidwillii Hook. & 1 & 0 & - & - & - & - & - & - & - \\
\hline Eucalyptus camaldulensis Dehn. & 21 & 57 & 92 & 0 & 8 & 25 & 75 & $(1-75)$ & $(51-75)$ \\
\hline Eucalyptus cinerea Mueller & 5 & 80 & 100 & 0 & 0 & 100 & 0 & $(1-25)$ & - \\
\hline Eucalyptus dunnii Maiden & 63 & 62 & 49 & 33 & 18 & 100 & 0 & $(1-50)$ & $(1-50)$ \\
\hline Eucalyptus globulus Labillardière & 15 & 0 & - & - & - & - & - & - & - \\
\hline Eucalyptus maidenii F.Muell. & 36 & 8 & 0 & 100 & 0 & 100 & 0 & - & $(6-10)$ \\
\hline Eucalyptus tereticornis Sm. & 12 & 17 & 0 & 100 & 0 & 50 & 50 & - & $(11-50)$ \\
\hline Eucalyptus viminalis Hook. & 3 & 0 & - & - & - & - & - & - & - \\
\hline Total Eucalyptus spp. & 155 & 38 & 57 & 30 & 13 & 83 & 17 & $(1-75)$ & $(1-75)$ \\
\hline Castanea sativa Mill. & 12 & 83 & 0 & 90 & 10 & 100 & 0 & $(11-25)$ & $(1-75)$ \\
\hline Casuarina sp. Miquel & 33 & 70 & 30 & 13 & 57 & 22 & 78 & $(1-50)$ & $(1-75)$ \\
\hline Cedrus deodara Loudon & 11 & 55 & 0 & 83 & 17 & 100 & 0 & $(1-5)$ & $(1-50)$ \\
\hline Celtis tala Gillies ex Planch. & 13 & 8 & 0 & 100 & 0 & 100 & 0 & - & $(1-5)$ \\
\hline $\begin{array}{l}\text { Cinnamomum glanduliferum } \\
\text { (Wall.) Meisn. }\end{array}$ & 8 & 0 & - & - & - & - & - & - & - \\
\hline Cupressus sp. L. & 12 & 33 & 100 & 0 & 0 & 100 & 0 & $(6-50)$ & - \\
\hline Fraxinus sp. L. & 19 & 63 & 50 & 0 & 50 & 92 & 8 & $(11-75)$ & $(51-100)$ \\
\hline Gleditsia triacanthos $\mathrm{L}$. & 37 & 24 & 56 & 22 & 22 & 100 & 0 & $(1-50)$ & $(1-75)$ \\
\hline $\begin{array}{l}\text { Ligustrum lucidum Buch.-Ham. } \\
\text { ex Wall. }\end{array}$ & 38 & 16 & 33 & 33 & 34 & 100 & 0 & $(6-50)$ & $(6-75)$ \\
\hline Morus nigra Trunb. & 1 & 100 & 0 & 0 & 100 & 100 & 0 & $(11-25)$ & $(51-75)$ \\
\hline Pinus elliottii Engelm. & 13 & 85 & 91 & 0 & 9 & 100 & 0 & $(1-50)$ & $(51-75)$ \\
\hline Populus spp. L. & 43 & 28 & 8 & 84 & 8 & 92 & 8 & $(11-75)$ & $(1-100)$ \\
\hline Quercus robur Asso. & 62 & 44 & 26 & 37 & 37 & 81 & 19 & $(1-75)$ & $(1-75)$ \\
\hline Robinia pseudoacacia L. & 18 & 22 & 0 & 100 & 0 & 100 & 0 & - & $(1-25)$ \\
\hline Salix babylonica $\mathrm{L}$. & 39 & 59 & 22 & 65 & 13 & 61 & 39 & $(1-25)$ & $(1-50)$ \\
\hline Salix sp. L. & 11 & 9 & 0 & 100 & 0 & 100 & 0 & - & $(26-50)$ \\
\hline Total Salix spp. & 50 & 48 & 21 & 67 & 12 & 62 & 38 & $(1-25)$ & $(1-50)$ \\
\hline Ulmus spp. L. & 16 & 69 & 0 & 46 & 54 & 100 & 0 & $(6-100)$ & $(6-75)$ \\
\hline
\end{tabular}


descortezados $(\overline{\mathrm{X}}=0,1950 \mathrm{~m})(P<0,001)$, sin diferencias significativas respecto a la altura (descortezados: $\overline{\mathrm{X}}=17,7$ m; no descortezados: $\overline{\mathbf{x}}=17,8 \mathrm{~m})(P=0,923)$.

\section{DISCUSIÓN}

Actividad de descortezado. Callosciurus erytrhaeus se comporta como una especie generalista en cuanto a las especies que daña, afectando un amplio espectro de árboles, además de otras plantas registradas en Argentina (Zubelzu et al. 2015). Las heridas fueron longitudinales o transversales en tronco y/o ramas, como fue descripto para esta y otras especies de ardillas (Hori et al. 2006, Mori et al. 2015), dependiendo de la especie arbórea considerada y de los motivos del descortezamiento, como ser obtener

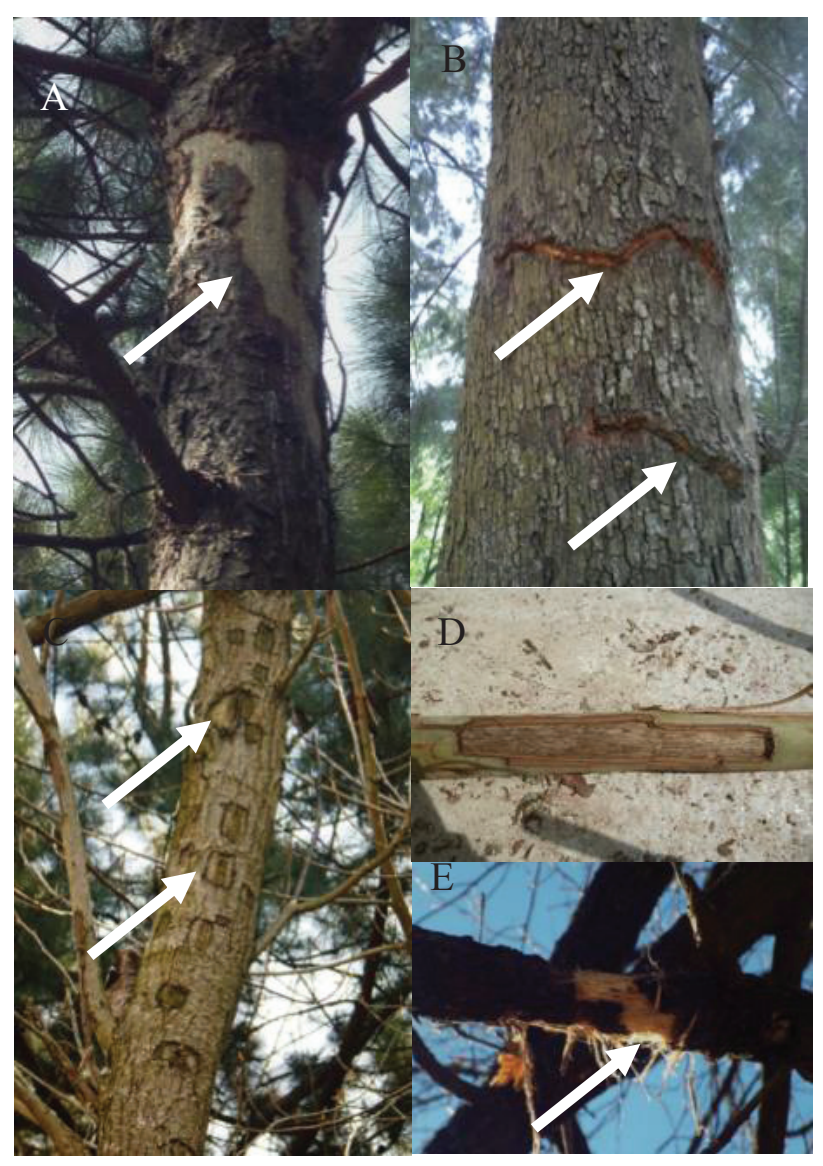

Figura 2. Heridas ocasionadas por la ardilla de vientre rojo. (A) Herida longitudinal en tronco de Pinus elliottii, (B) heridas transversales en tronco de Casuarina sp., (C) heridas longitudinales en tronco de Populus spp., (D) herida longitudinal en rama de Eucalyptus sp., (E) herida longitudinal con deshilachado en rama de Quercus robur. Las flechas señalan la ubicación de las heridas.

Wounds caused by red-bellied squirrel. (A) Longitudinal wound in trunk of Pinus elliottii, (B) cross wounds in trunk of Casuarina sp., (C) longitudinal wounds in trunk of Populus spp., (D) longitudinal wound in branch of Eucalyptus sp., (E) longitudinal frayed wound in branches of Quercus robur. Arrows indicate location of damage. material para la confección del interior de los nidos o la obtención de savia (Tamura y Ohara 2005).

Los árboles dañados presentaron un mayor DAP que los no dañados, por lo cual C. erytrhaeus podría representar una mayor amenaza con respecto a otros pequeños mamíferos, como fue postulado por Sullivan et al. (1993). En otros estudios se ha encontrado que las ardillas suelen descortezar árboles con edades intermedias, quizás relacionado a los grandes diámetros de los árboles muy añejos (Mayle et al. 2009). Son necesarios análisis complementarios para evaluar la selección de tamaños o cortezas arbóreas determinadas por parte de las ardillas.

Implicancias de la altura e intensidad de daño. Varias de las especies dañadas son de interés productivo forestal (eucaliptos, sauces, álamos y coníferas) y de uso agronómico u ornamental (robles y casuarinas). En este sentido, la altura del daño en los troncos, la proporción del tronco afectado (intensidad de daño) y la profundidad de las heridas, son factores importantes debido al potencial deterioro de la calidad de la madera de las especies forestales, pudiendo ser más graves las consecuencias cuando la madera se destina a usos sólidos (aserrío, debobinado).

Eucalyptus dunnii, una de las especies de eucaliptos más utilizada en la Argentina para uso de molienda, presenta daño en el fuste, con un $64 \%$ de individuos con heridas por debajo de los $3 \mathrm{~m}$ de altura; se observaron además rebrotes y quebraduras, daño que podría tener graves consecuencias para el aprovechamiento de la madera, si bien esto no ha sido cuantificado en este trabajo. El daño ocasionado por las ardillas podría generar un debilitamiento inicial que deja a los árboles más susceptibles a roturas ocasionadas por el viento y a infecciones secundarias causadas por hongos u otros patógenos (Zhu et al. 1990).

En E. tereticornis y E. camaldulensis, en salicáceas y en P. elliottii se encuentran individuos con altas intensidades de daño (entre $50 \%$ y $75 \%$ del tronco o rama afectado); también se observa anillado y descope de individuos lo que podría tener un impacto negativo potencial en la sobrevida y calidad de esos individuos u obligar al productor a dirigir el producto sólo a molienda (Sullivan et al. 1993, Richter 2015), si bien es preciso cuantificar las pérdidas económicas. En el caso de Q. robur, en Argentina es una especie de importancia ornamental, siendo escasos los rodales dedicados a la producción; sin embargo, una de las áreas evaluadas pertenece a un rodal productivo por lo que el daño ocasionado podría afectar su calidad limitando su uso para aserrío.

Papel de la ardilla como potencial plaga forestal. Los resultados del presente trabajo indican que puede considerarse a la ardilla de vientre rojo como una especie dañina o perjudicial en su área de influencia, ya que ocasiona daños puntuales en plantaciones forestales. Teniendo en cuenta que estudios previos en Argentina muestran que presenta altas densidades (Benitez et al. 2013), que continúa dispersándose por causas naturales y antrópicas, y que carece de 
depredadores naturales que puedan mitigar sus poblaciones, podría en algún momento alcanzar la "condición de plaga" (Monge 2007) y ser una amenaza para otras regiones forestales del país si continúa expandiéndose.

Este trabajo brinda información preliminar sobre aspectos cuantificables del daño ocasionado por C. erytrhaeus en las especies forestales presentes en el principal foco de invasión en Argentina. Futuros estudios que analicen los tejidos afectados y las pérdidas de volumen de madera permitirán evaluar la magnitud y consecuencias del daño en términos económicos y su implicancia para las producciones comerciales.

\section{AGRADECIMIENTOS}

A las instituciones que permitieron el ingreso a sus establecimientos, Pamela Zubelzu, Borja Baguette Pereiro, Verónica Benitez y Cecilia Gozzi. Este trabajo fue financiado con un subsidio y Beca de Investigación otorgado por la Universidad Nacional de Luján.
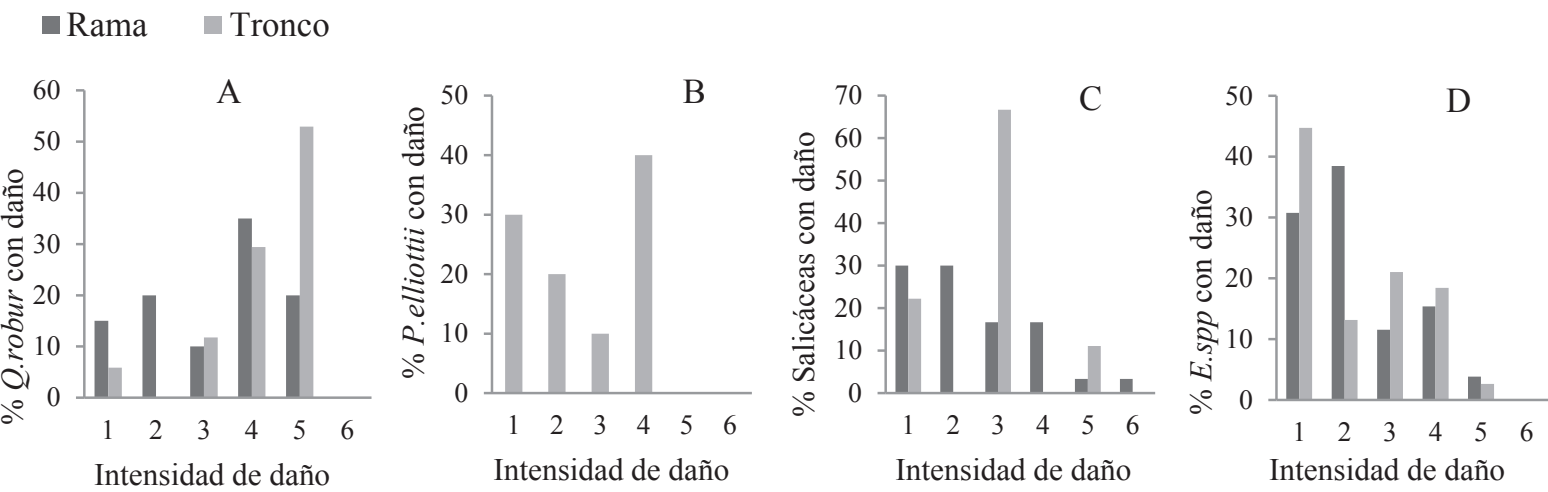

Figura 3. Porcentaje de árboles dañados en tronco y rama de (A) Quercus robur, (B) Pinus elliottii, (C) Salicaceae y (D) Eucalyptus spp., según las siguientes categorías: 1: (1-5\%), 2: (6-10\%), 3: (11-25\%), 4: (26-50 \%), 5: (51-75\%), 6 (76-100 \%).

Percentage of debarked poles and/or branches on (A) Quercus robur, (B) Pinus elliottii, (C) Salicaceae, and Eucalyptus spp. trees, according to different categories: (1: (1-5\%), 2 (6-10\%), 3: (11-25\%), 4: (26-50\%), 5 (51-75\%), 6 (76-100 \%)).
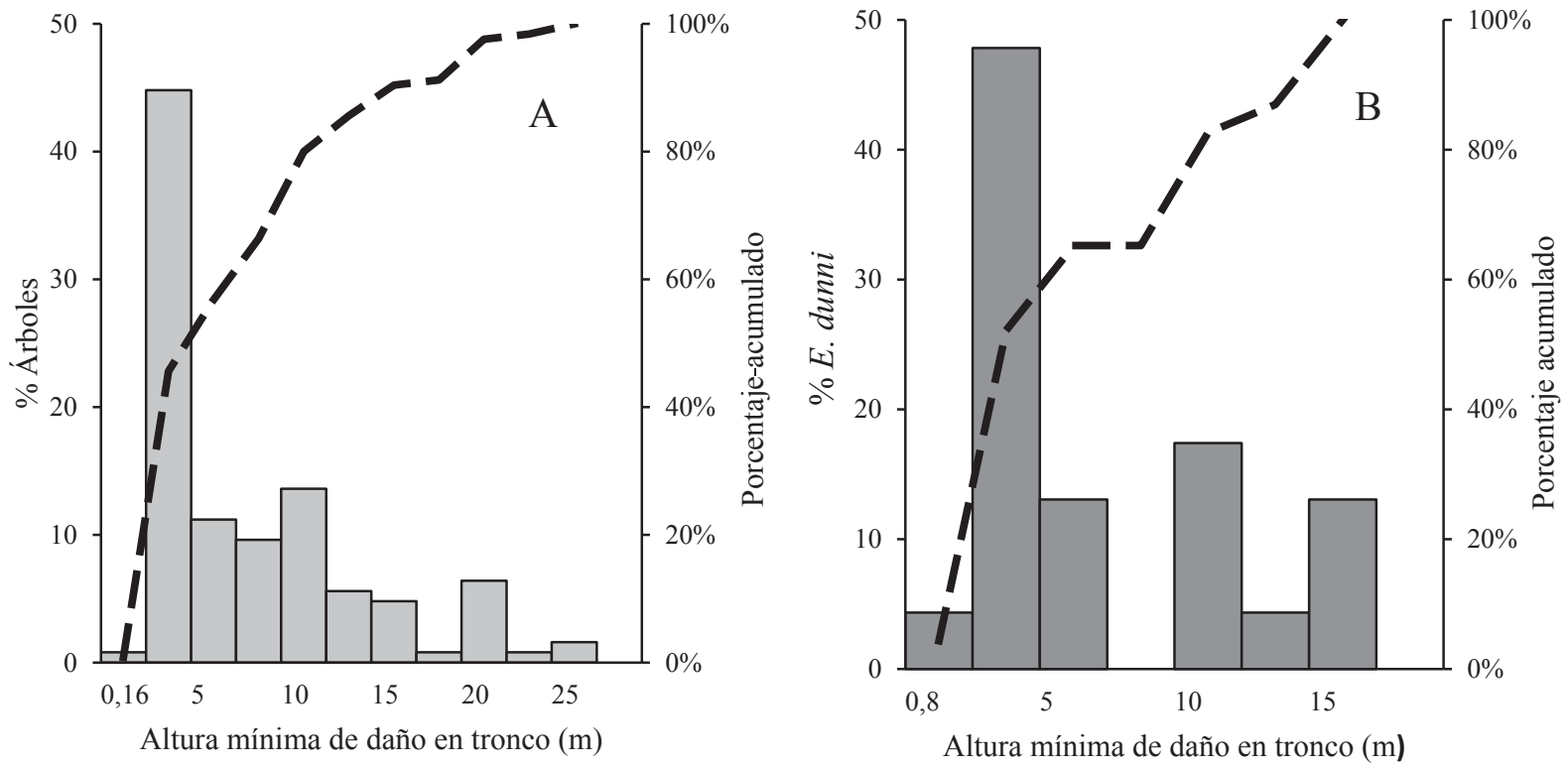

Figura 4. Porcentaje de individuos con tronco descortezado a distintas clases de altura mínima, considerando (A) todos los árboles muestreados y (B) Eucalyptus dunnii. La línea punteada indica el porcentaje acumulado.

Percentage of debarked trees at minimum height classes considering (A) all individuals and (B) Eucalyptus dunnii. The dotted lines indicate the cumulative percentage. 


\section{REFERENCIAS}

Aprile G, D Chicco. 1999. Nueva especie exótica de mamífero en la Argentina: la ardilla de vientre rojo (Callosciurus erythraeus). Mastozoología Neotropical 6(1): 7-14.

Benitez VV, S Almada Chavez, AC Gozzi, ML Messetta, ML Guichón. 2013. Invasion status of Asiatic red-bellied squirrels in Argentina. Mammalian Biology 78: 164-170.

Bertolino S, P Lurz. 2013. Callosciurus squirrels: worldwide introductions, ecological impacts and recommendations to prevent the establishment of new invasive populations. Mammalian review 43: 22-33.

Gozzi AC, ML Guichón, VV Benitez, GN Romero, C Auteri, B Brihuega. 2013. First isolation of Leptospira interrogans from the introduced arboreal squirrel Callosciurus erythraeus (Rodentia: Sciuridae) introduced in Argentina. Wildlife Biology 19(4): 483-489.

Guichón ML, M Bello, L Fasola. 2005. Expansión poblacional de una especie introducida en la Argentina: la ardilla de vientre rojo Callosciurus erythraeus. Mastozoología Neotropical 12: 189-197.

Guichón ML, VV Benitez, AC Gozzi, M Hertzriken, M Borgnia. 2015. From a lag in vector activity to a constant increase of translocations: invasion of Callosciurus squirrels in Argentina. Biological Invasions 17(9): 2597-2604.

Hori M, M Yamada, N Tsunoda. 2006. Line census and gnawing damage of introduced Formosan squirrels (Callosciurus erythraeus taiwanensis) in urban forests of Kamakura, Kanagawa, Japan. In Koike F, MN Clout, M Kawamichi, M de Poorter, K Iwatsuki eds. Assessment and Control of Biological Invasion Risks. Kyoto, Japan. Shoukadoh. p. 204-209.

Mayle B, Proudfoot J, Poole J. 2009. Influence of tree size and dominance on incidence of bark stripping by grey squirrels to oak and impact on tree growth. Forestry 82 (4): 431-444.

Messetta ML, FA Milesi, ML Guichon. 2015. Impacto de la ardilla de vientre rojo sobre la comunidad de aves en la Región Pampeana, Argentina. Ecología Austral 25: 37-45.

Monge J. 2007. ¿Qué son las plagas vertebradas? Agronomía Costarricense 31(2): 111-121.
Mora-Ascencio P, A Mendoza-Durán, C Chávez. 2010. Densidad poblacional y daños ocasionados por la ardilla Sciurus aureogaster: implicaciones para la conservación de los viveros de Coyoacán, México. Revista Mexicana de Mastozoología 14: 7-22.

Mori E, PJ Mazzoglio, PC Rima, G Aloise, S Bertolino. 2015. Bark-stripping damage by Callosciurus finlaysonii introduced into Italy. Mammalia 80(5): 507-514.8 p. DOI: 10.1515/ mammalia-2015-0107.

Núñez CE. 2007. Morfología Fibrosa de la Madera del Eucalyptus Grandis Implantado en La Mesopotamia Argentina. 1. Atributos biométricos. Revista Ciencia y Tecnología 9(9): 36-43.

Richter C. 2015. Vertebrates: Browsing, fraying, and stripping damage. In Richter C ed. Wood Characteristics. Description, Causes, Prevention, Impact on Use and Technological Adaptation. Cham, Switzerland. Springer. p. 139-147. DOI 10.1007/978-3-319-07422-1.

Smallwood KS, TP Salmon. 1992. A rating system for potential exotic vertebrate pests. Biological Conservation 62: 149159.

Sullivan TD, H Coates, LA Jozsa, PK Diggle. 1993. Influence of feeding damage by small mammals on tree growth and wood quality in young lodgepole pine. Canadian Journal of Forest Research 23 (5): 799-809.

Tamura N, Ohara S. 2005. Chemical components of hardwood barks stripped by the alien squirrel Callosciurus erythraeus in Japan. Journal of Forestry Research 10: 429-433.

Yin S, Z Wen, J Ran, K Tang. 2010. Damage of Callosciurus erythraeus in Planted Forests. Sichuan Journal of Zoology 3: 69-76.

Zhu Y, W Zhang, X Zhu. 1990. Bark-stripping damage to forest trees by red-bellied tree squirrel (Callosciurus erythraeus) in Zhejiang Province. Acta Theriologica 10: 276-281.

Zubelzu P, M Hertzriken, V Benitez y M Borgnia. 2015. Ardillas de vientre rojo: descortezado de la comunidad arbórea ocasionado por esta especie introducida. In II Jornadas Patagónicas de Ciencias Ambientales. Trelew, Chubut, Argentina. 23-25 septiembre. s/p. 